\title{
L'armée, livre des hommes : passeurs de symboles et personnages liminaires dans Servitude et grandeur militaires d'Alfred de Vigny
}

Alfred de Vigny a souvent projeté une image d'écrivain philosophe et ses préfaces y sont pour beaucoup. En 1827 par exemple, dans les "Réflexions sur la vérité dans l'art » qui introduisent le roman Cinq-Mars, Vigny admet vouloir représenter " le spectacle philosophique de l'homme profondément travaillé par les passions de son caractère et de son temps" $(1993$, p. 8$)$. On retrouve un constat similaire dans la préface de 1837 des Poèmes antiques et modernes, où il indique que, dans ses œuvres, "une pensée philosophique est mise en scène sous une forme Épique ou Dramatique » (1986, p. 5). Même si le mot "philosophique " n'a pas toujours le même sens chez Vigny et « s'éclaire par contraste " (Jarry, 1993, p. 181), la critique a eu tendance à voir dans ses œuvres des écrits à thèse : «En privilégiant la pensée du poète philosophe, toute une tradition de la critique vignyenne a ainsi eu tendance à niveler la variété de ses créations, les ramenant à un ensemble d'idées inlassablement déclinées " (Hautbout et Melmoux-Montaubain, 2010, p. 13).

Par contre, cela n'empêche pas de retrouver des enseignements ou des figures de maître dans les écrits du poète romantique. Ils prennent simplement des formes plus subtiles. Pour Vigny, l'écriture doit structurer une expérience qui fera naître une idée chez le lecteur :

Jordan Diaz-Brosseau - doctorant en cotutelle à l'Université du Québec à Montréal et à l'Université de Lorraine. Adresse de correspondance : 4 rue de Pont-à-Mousson, 57000, Metz, France, e-mail : diaz-brosseau.jordan@courrier.uqam.ca

ORCID iD : https://orcid.org/0000-0002-8504-3768 
dans Servitude et grandeur militaires, on repère ainsi toute une réflexion sur l'enseignement, condamnant " tous les conseils écrits ou parlés », les sermons et le «langage froid et pédantesque » des livres parce que, selon le capitaine Renaud, «l'expérience seule et le raisonnement qui sort de nos propres réflexions peuvent nous instruire ». Cette affirmation peut s'entendre comme une valorisation de l'ouvrage de Vigny, en ce que chacun des témoignages des soldats rapporte justement une expérience que le lecteur est conduit à méditer avec profit. (Hautbout, 2010, p. 211)

Si maître il y a chez Vigny, ce n'est pas une figure de professeur transmettant un savoir à son élève. La vérité chez lui se présente sous la forme d'un « Drame de la pensée » et « le seul maître c'est celui qui daigne faire descendre dans l'homme l'émotion féconde, et faire sortir les idées de nos fronts, qui en sont brisés quelquefois » (1986, p. 758), comme il l'écrit dans la «Dernière nuit de travail » de Chatterton (1834). Ce type de savoir prend la forme d'une maïeutique. Il faut le dire, nous n'entendons pas ce terme comme Marc Citoleux qui, lisant Stello (1832), le définit comme

l'art de conduire les disciples à leur insu et souvent malgré eux où l'on veut et pour leur bien. Le docteur Noir veut guérir Stello et l'acheminer à cette constatation que la société maudit les poètes et les écarte. [...] La maïeutique tend à la définition. La définition dégage des faits l'idée générale. C’est ainsi que le docteur Noir dégage des trois aventures de Gilbert, de Chatterton et de Chénier trois maximes. (1924, p. 505-506)

Il est vrai que certains personnages répondent parfois à cette ambition, mais ce serait passer rapidement sur la part d'activité critique exigée du lectorat et que favorise le texte par l'expérience formative qu'il offre. Un passage comme celui du seizième chapitre de Stello, où le Docteur Noir se plaint d'être " retombé dans l'état fâcheux d'un homme forcé de causer au lieu de prêcher » (Vigny, 1993, p. 605), invite à nuancer l'analyse de Citoleux. Les apories vignyennes, parfois impossibles à trancher, ont surtout une visée réflexive. Elles permettent au romancier d’élaborer une «philosophie de la relation » (Jarry, 1993, p. 194) qui conduit à appréhender le lecteur comme un agent actif que le texte «met au travail» (Hautbout, 2010, p. 214). Vigny ne fournit qu'une des deux pièces à une construction qui se " déroule à présent entre deux instances pareillement actives : l'auteur qui "aime" ; la "Postérité" qui "regarde" " (Jarry, 1993, p. 194). En ce sens, le maître prend la forme d'un passeur de symbole et d'un initiateur : il offre des fragments de vérités que le disciple réorganise selon ses besoins. Il y a transferts et dialogues entre le maître et l'élève, entre le poète et le lecteur, car le

symbole n'est pas figé, il n'est pas une clef forgée pour donner accès à un sens tapi dans les antichambres imagées de la conscience. Il est un interprétant que l'écrivain induit avec son lectorat. Et cette relation ne cesse d'être labile du fait de l'évolution de ce lectorat qui, en chaque point du déroulement de l'histoire, va trouver dans l'œuvre de l'écrivain la moitié congruente du sens partiel qu'il détient en raison de ses intérêts particuliers, lesquels sont évidemment propres à chaque époque (Saint-Gérand, 2016, p. 114). 
Nous proposons d'étudier ce procédé au travers d'une lecture de Servitude et grandeur militaires (1835). Pour ce faire, nous insisterons plus particulièrement sur la structuration d'une pédagogie de l'expérience par l'architecture de l'ouvrage. Celle-ci s'organise dialectiquement et chaque palier supérieur intègre la contradiction de la relation précédente pour l'amener à un autre niveau. Nous verrons que le narrateur qui écoute les récits est conduit à être redoublé par le lecteur qui lit les nouvelles écrites, alors même que le savoir transmis au premier découle directement d'un conflit entre l'écriture et l'oralité. Nous privilégierons une lecture anthropologique et ethnocritique du récit, alliant " poétique du littéraire » et " ethnologie du symbolique " (Cnockaert, Privat, Scarpa, 2011, p. 5), avec pour objectif d'analyser les mécanismes de socialisation proposés, en premier lieu, au jeune narrateur par le biais de ses maitres à penser, et, ensuite, au lecteur au travers des formes narratives de l'expérience. Si le récit se présente comme un rite initiatique qui cherche à s'accomplir sur plusieurs niveaux, alors ses ressorts aident à voir comment Vigny, en passeur de symboles, façonne un apprentissage qu'il invite ensuite à dépasser.

\section{Un texte à triple fond}

Vigny fut militaire entre 1814 et 1827. Servitude et grandeur militaires est son dernier roman achevé et il y consigne des histoires entendues pendant son service. Le roman est composé de trois récits parus individuellement dans la Revue des deux mondes avant d'être refondus en un seul livre en 1835 : "Laurette ou le Cachet rouge " paraît le $1^{\text {er }}$ mars 1833 ; «La Veillée de Vincennes », le $1^{\text {er }}$ avril 1834 ; et « La Vie et la Mort du Capitaine Renaud ou Canne de Jonc », le $1^{\text {er }}$ octobre 1835, aussitôt suivi de l'édition livresque dans laquelle l'auteur développe un essai qui sert « d'enveloppe aux trois nouvelles, et fait de l'ouvrage un livre à triple fond» (Jarry, 2010, p. 84). À cheval entre un recueil de nouvelles et un roman, le statut générique de ce texte est encore très discuté.

L'argumentaire du texte peut paraître simple : un narrateur-écrivain extradiégétique expose l'histoire de sa désillusion face à l'armée. Il repasse alors à travers certains souvenirs pour retracer le parcours de sa "lente guérison pour cette maladie de l'enthousiasme militaire " (Vigny, 1993, p. 721). Dans les trois cas, le jeune narrateur homodiégétique effectue la rencontre d'un soldat plus âgé qui lui contera ses aventures. Dans la première nouvelle, le narrateur rencontre un ancien capitaine, innomé, qui lui confiera comment il reçut l'ordre, par le biais d'une lettre officielle du Directoire, d'exécuter un jeune homme qu'il devait déporter au bagne de Cayenne et pour qui il avait beaucoup de sympathie. Ce récit raconte le drame d'un marin dont l'abnégation fut poussée trop loin et dont les regrets sont symbolisés par la prise en charge de Laurette, la fiancée devenue folle du jeune homme. Le second récit met en scène un adjudant trop scrupuleux qui, la veille d'une inspection officielle, fait accidentellement exploser le stock de poudre dont il a la charge. Avant le drame, 
lors d'une veillée, l'adjudant Mathurin raconte au jeune narrateur comment il obtint de Marie-Antoinette la possibilité d'épouser Pierrette, sa défunte femme au moment du récit. Ce souvenir justifie ainsi le très (trop) haut degré d'honneur qui pousse le soldat à terminer son inspection hors des heures d'ouverture, amenant ainsi avec lui une flamme pour s'éclairer dans un lieu rempli d'explosifs. Le dernier récit est celui du capitaine Renaud, alias Canne-de-Jonc, un officier qui relate cette fois comment, enfant, il rencontre Napoléon et comment, en vieillissant, il apprend à relativiser l'amour immodéré qu'il a développé pour son chef de guerre. Il confie notamment au narrateur les circonstances qui l'ont amené à tuer un enfant pendant sa garde en Russie ainsi que les circonstances de sa captivité sur le navire anglais du commandant Collingwood.

Ces récits tragiques servent à illustrer la condition désolante des soldats et Vigny effectue des rapprochements entre cette réalité et celle du poète. Il écrit en conclusion du roman : "Après avoir, sous plusieurs formes, expliqué la nature et plaint la condition du Poète dans notre société, j’ai voulu montrer ici celle du Soldat, autre Paria moderne » (1993, p. 820). Ce faisant, comme pour le poète dans Stello, il s'agit de montrer que l'homme de guerre est une victime et que son intégration sociale est désormais devenue difficile, voire impossible.

\section{Roman de la désillusion et initiation ratée}

Historiquement parlant, l'armée est moins mobilisée lors de la Restauration que sous le Directoire ou pendant le Premier Empire. C'est pourquoi le narrateur extradiégétique précise : "Au moment où jécris, un homme de vingt ans de service n'a pas vu une bataille rangée. J'ai peu d'aventures à vous raconter, mais j'en ai entendu beaucoup " (Vigny, 1993, p. 684). Cette oisiveté lui fera voir à quel point l'armée, en temps de paix, est une institution «barbare et arriérée » (1993, p. 683), notamment parce que les militaires ne s'insèrent plus dans la société comme le faisaient auparavant les citoyens-soldats durant les grandes heures de l'Antiquité. Il le dit bien : "L'armée est une nation dans une nation ; c'est un vice de nos temps. Dans l'Antiquité il en était autrement : tout citoyen était guerrier, et tout guerrier était citoyen» (1993, p. 689).

Les reproches du narrateur visent surtout les hommes qui ont contribué à l'exaltation militaire des jeunes garçons. Il explique appartenir «à cette génération née avec le siècle, qui, nourrie de bulletins par l'Empereur, avait toujours devant les yeux une épée nue, et vint la prendre au moment même où la France la remettait dans le fourreau des Bourbons " (1993, p. 684). Ce faisant, il lui prit « un amour vraiment désordonné de la gloire des armes » (1993, p. 688). Ces blâmes concernent aussi son père. Dès le début du roman, le narrateur est explicite sur l'influence des récits paternels :

J'aimai toujours à écouter, et quand jétais tout enfant, je pris de bonne heure ce goût sur

les genoux blessés de mon vieux père. Il me nourrit d'abord de l'histoire de ses campagnes, 
et, sur ses genoux, je trouvai la guerre assise à côté de moi ; il me montra la guerre dans ses blessures, la guerre dans les parchemins et le blason de ses pères, la guerre dans leurs grands portraits cuirassés, suspendus, en Beauce, dans un vieux château. Je vis dans la Noblesse une grande famille de soldats héréditaires, et je ne pensai plus quà m'élever à la taille d'un soldat. (1993, p. 685-686)

La marginalité sociale des militaires à partir de la Restauration n'empêche pas que le service soit demeuré un rite de passage durablement inscrit dans l'imaginaire des jeunes hommes. André Rauch explique comment

il devient pour les citoyens de sexe masculin un passage obligé de la jeunesse à la vie adulte. La levée en masse, inconnue jusque-là, encore étrangère aux royaumes d'Europe, transforme la condition masculine. Ce qui avait été le métier de quelques-uns - porter les armes - devient la caractéristique de tous, une qualité qui distingue l'homme de l'enfant, de la femme, mais aussi de l'infirme et du vieillard. (2000, p. 48)

De même, Anne-Marie Sohn constate que " l'électeur et le soldat constituent deux pôles de la masculinité publique et politique. La figure du volontaire de 1792 et le "mythe du grognard" hantent jusquà la Troisième République l'imaginaire de la jeunesse masculine » $(2009$, p. 181).

Les premiers fantasmes de l'enfant sont donc déçus en ce que l'armée ne permet pas l'initiation tant espérée. Non seulement elle ne civilise pas, mais elle ensauvage :

Ainsi la guerre s'est civilisée, mais non les armées ; car non seulement la routine de nos coutumes leur a conservé tout ce qu'il y avait de mauvais en elles ; mais l'ambition et les terreurs des gouvernements ont accru le mal, en les séparant chaque jour du pays, et en leur faisant vivre une servitude plus oisive et plus grossière que jamais. (Vigny, 1993, p. 684)

Nous pouvons ainsi admettre que « le fait d'adopter le père comme modèle, figure à imiter, condamne a priori in ovo le fils à lui ressembler et donc à échouer dans ce vain effort » (Ozwald, 2010, p. 133). Ce désappointement explique pourquoi le roman peut être est qualifié, comme le fait André Jarry, "de désillusion de l'épopée » (1998, p. 224). Vigny lui-même le qualifie de « sorte de poème épique sur la désillusion » dans son Journal (1949, p. 1037).

\section{Personnages liminaires, figures de passeurs et rites de passage}

Il convient alors d'analyser comment se surmonte la désillusion. La polyphonie narrative ne permet pas tellement d'y répondre, d'autant plus qu'elle infirme parfois ce que valorise la partie essayistique. Vigny par exemple y défend une « Religion de l'hon- 
neur » (1993, p. 825) alors même que le récit de «Laurette » montre les conséquences d'une telle logique lorsqu'elle devient trop radicale. Par contre, la maîtrise des savoirs narratifs représente une voie féconde pour comprendre comment le narrateur revient sur sa propre condition et la dépasse. Le ratage initial contraint le narrateur à se trouver de nouvelles figures d'autorité. Les grognards bénéficieront d'une certaine indulgence puisque ce sont leurs récits formateurs qui apparaîtront comme un remède au fantasme guerrier hérité des pères et de l'Empire. Par exemple, suite à la l'écoute de l'histoire de « Laurette», le romancier écrit :

Je me souviens de la consternation que cette histoire jeta dans mon âme ; ce fut peutêtre là le principe de ma lente guérison, pour cette maladie de l'enthousiasme militaire. Je me sentis tout à coup humilié de courir des chances de crimes, et de me trouver à la main un sabre d'Esclave au lieu d'une épée de Chevalier. (1993, p. 721)

De même, après l'histoire de l'adjudant de la «Veillée », le narrateur dit: «Combien de fois ce vieux soldat vaut-il mieux avec sa résignation, que nous autres, jeunes officiers, avec nos ambitions folles! Cela nous donna à penser» (1993, p. 755).

Ces êtres exemplaires représentent les premiers maitres rencontrés dans le roman. Ce sont des passeurs ou ce que Marie Scarpa nomme des personnages liminaires. Pour Scarpa, le personnage liminaire est,

selon les circonstances et les contextes, non initié, mal initié ou sur-initié (voire le tout en même temps). Il est placé, dans le système des normes culturelles, du côté du pôle le moins positif ou le plus problématique. Fréquemment, il est un personnage secondaire, qui sert alors de valeur ou de contre-valeur témoin dans le système du personnel romanesque. (2011, p. 181)

Ce type de personnage est un vecteur de narrativité en ce qu'il constitue un pôle d'altérité à partir duquel se construisent le protagoniste et l'écriture romanesque. Vigny ne cache d'ailleurs pas que l'ouvrage est composé " plutôt par les mémoires des autres que des [s]iens » (1993, p. 684). Le personnage principal réussit son intégration sociale, mais ce sera à la fois grâce et au détriment des trois personnages initiateurs qui mourront repoussés en marge de la cité à la fin de chaque récit (le premier meurt à Waterloo (1993, p. 720); le second, de l'explosion qu'il provoque (1993, p. 760) ; et le troisième est assassiné par un enfant pendant la révolution de 1830 (1993, p. 819).

$\mathrm{Si}$ « le rite permet de "mesurer" le type de socialisation (en termes d'intégration, d'autonomisation, etc.) » (Scarpa, 2011, p. 179), le degré de maîtrise des savoirs narratifs nous apparait être un marqueur témoignant du niveau de réussite du rite. Un commentaire métanarratif comme celui de l'adjudant Mathurin nous semble illustrer parfaitement cette logique du roman : « Si je savais faire des surprises, mon lieutenant, comme on en fait dans les livres, [...] je trouverais une manière nouvelle 
de vous dire la suite de ceci; mais je vais de fil en aiguille, tout simplement comme a été ma vie de jour en jour [...] » (Vigny, 1993, p. 749). L'impossibilité du livre témoigne du seuil de socialisation et annonce la marge où se trouvera finalement repoussé ce conteur.

Étienne Kern voit trois phases dans le roman : l'une correspond à l'enfance et au foyer paternel ; la seconde, à l'adolescence qui se déroule entre l'école et l'armée ; et la troisième, à la maturité de l'écriture romanesque (2010, p. 165-166). Évidemment, seul le narrateur atteint le troisième moment. Nous adhérons à cette tripartition qui explique bien les différents niveaux narratifs de l'œuvre, mais aussi parce qu'elle redouble la forme anthropologique du rite de passage. Arnold Van Gennep, dans son célèbre livre sur Les Rites de passage, divise lui aussi en trois phases ce genre de ritualité : la phase préliminaire, la phase de marge et l'agrégation (1981). Nous postulons ainsi une homologie entre le rite et le récit, puisque l'armée est justement un lieu de marge, entre le foyer et l'agora, où "l'individu s'expérimente autre pour devenir soi dans un nouveau statut» (Scarpa, 2011, p. 180).

L’armée, depuis l'Antiquité au moins, joue un rôle d'hétérotopie de crise, c'està-dire qu'elle fournit un « lieu autre " pour les périodes de transition comme celles de la puberté masculine : « le service militaire pour les garçons a joué certainement un tel rôle, les premières manifestations de la sexualité virile devant avoir lieu précisément "ailleurs" que dans la famille " (Foucault, 2001, p. 1576). Le problème, à lépoque de Vigny, c'est que l'institution est plus de l'ordre d'une hétérotopie de déviation, " celle dans laquelle on place les individus dont le comportement est déviant par rapport à la moyenne ou à la norme exigée » (p. 1576). Elle n'autorise plus l'agrégation sociale à laquelle elle doit mener. Pourtant, même s'il s'agit d'un modèle perdurant dans une société qui cherche à s'en détourner, l'armée peut encore servir cette fonction aux yeux de Vigny, à condition d'en changer les modalités. Les personnages liminaires conservent leur statut de passeurs, mais Vigny, plus lucide sur l'histoire et sa modernité, invite à actualiser leurs enseignements et l'écriture romanesque semble être la voie privilégiée pour civiliser ces mœurs. Il cherchera à mettre ce savoir à l'écrit parce que

l'armée est un bon livre à ouvrir pour connaître l'humanité; on y apprend à mettre la main à tout, aux choses les plus basses comme au plus élevées; les plus délicats et les plus riches sont forcés de voir de près la pauvreté et de vivre avec elle, de lui mesurer son gros pain et de lui peser sa viande. Sans l’armée, tel fils de grand seigneur ne soupçonnerait pas comment un soldat vit, grandit, engraisse toute l'année avec neuf sous par jour et une cruche d'eau fraîche, portant sur le dos un sac dont le contenant et le contenu coûtent quarante francs à sa patrie. (Vigny, 1993, p. 725)

Il semble alors que le terme " livre " peut être pris au premier degré ou être lu comme une métaphore dans ce cas précis. 


\section{Oralité et écriture}

Le romancier insiste sur le fait que la vie silencieuse de la caserne est au fondement même de son écriture. Dans sa préface aux Poèmes antiques et modernes, Vigny introduit ses poèmes en précisant qu'ils sont « ceux choisis par l'auteur parmi ceux qu'il composa pendant sa vie errante et militaire " (1986, p. 5). Dans une scène de "La Veillée de Vincennes ", le narrateur raconte comment "le masque de fer " des soldats lui inspire "La Prison» (Vigny, 1993, p. 729), un des poèmes de ce même recueil. Il acquiert un langage et un savoir à partir de l'expérience du camp : « De ces nuits, où j'agrandis en silence ce que j'avais reçu de savoir de nos études tumultueuses et publiques, sortirent mes poèmes et mes livres; de ces journées il me reste ces souvenirs dont je rassemble ici, autour d'une idée, les traits principaux » (Vigny, 1993, p. 689).

Le drame de la pensée vignyenne est alors de concilier l'écriture avec ce savoir, et ainsi redoubler le rite par le récit. La poétique de Vigny dépasse, dans un premier temps, cette contradiction en réconciliant les deux modalités par un acte polylogique qui combine les «modes de coalescences du logos de la raison graphique, et du logos de la parole vivante " (Privat, 2017, p. 63). Pour ce faire, le romancier expose toute une anthropologie de la caserne et livre ses codes et ses symboles. Il développe alors une scénographie de la parole vive et de la coutume orale dans le premier niveau de l'architecture textuelle. L'armée étant une institution fermée, le narrateur devient un locuteur privilégié. Vigny précise qu' « il n'y a pas de profession où la froideur des formes du langage et des habitudes contraste plus vivement avec l'activité de la vie que la profession des armes » (1993, p. 767). Il explique aussi que

l'autorité absolue qu'exerce un homme le contraint à une perpétuelle réserve. Il ne peut dérider son front devant ses inférieurs, sans leur laisser prendre une familiarité qui porte atteinte à son pouvoir. Il se retranche l'abandon et la causerie amicales, de peur qu'on prenne acte contre lui de quelque aveu de la vie ou de quelque faiblesse qui serait de mauvais exemple. J'ai connu des officiers qui s'enfermaient dans un silence de trappiste, et dont la bouche sérieuse ne soulevait la moustache que pour laisser passage à un commandement (Vigny, 1993, p. 694).

Le capitaine Renaud sied tout à fait à cette description. Le romancier l'introduit en soulignant qu'il ne " parlait que le moins possible et par monosyllabes " (Vigny, 1993, p. 765). Sachant cela, le narrateur s'étonne d'autant plus d'avoir une conversation avec lui. Le capitaine innomé de "Laurette » est lui aussi décrit comme un amateur de « silence et d'ordre » (1993, p. 706), et c'est bien parce que le jeune narrateur marche "sans rien dire " (1993, p. 699), "s'étonne bien peu» (1993, p. 700) et ne parle « que gravement et avec simplicité de [l']équipage, dont [le capitaine] craignait le ridicule " (1993, p. 700) que l'ancien marin "se mit à son aise tout d'un coup " (1993, p. 700). Comme les grognards recherchent « une patience égale à la leur et un 
silence aussi sérieux » (1993, p. 695) les recrues doivent être méritantes. Rauch a bien démontré qu'il y a là toute une dialectique entre le maître et la recrue :

Une reconnaissance réciproque entre conscrits et grognards (ceux que dans la Garde on appelle "les messieurs") fonde dans les armées de l'Empire un attachement essentiel à la dignité masculine ; la différence d'expérience établit entre eux un rapport d'inégalité, au moins de l'ordre de celui qui existe entre un cadet et son aîné. S’appuyant sur ce besoin d'émulation, avec cette nuance de paternité spirituelle ("le pauvre enfant") qui convient, le grognard a intégré les bonnes dispositions du cadet. L'ancien sent monter en lui une générosité dans laquelle une paternité spirituelle se crée : il s'instaure maitre en s'appuyant sur le noble besoin d'émulation de la jeune recrue. (2000, p. 70)

Lorsque Vigny décrit la religion de l'Honneur, il précise bien qu'il s'agit d'une " religion mâle, sans symbole et sans images, sans dogme et sans cérémonies, dont les lois ne sont écrites nulle part "(1993, p. 823, nous soulignons). Il est alors aisé de comprendre pourquoi le capitaine du brick, dans « Laurette », est mal à l'aise face à la lettre d'exécution, même si "l'arrêt de mort est en règle, et l'ordre d'exécution signé, paraphé, scellé » (1993, p. 711) : c'est parce qu'il « doit obéir à un morceau de papier ! car ce n'était que cela enfin!» (1993, p. 713). La littératie administrative agit comme un disjonctif social au sein de cette institution organisée essentiellement autour de rapports oraux. Et pourtant, l'enseignement vignyen passe par la domestication de cette pensée via l'écriture. Même si le poète retient la leçon, le passage de l'oralité vers l'écrit en représente le premier dépassement.

\section{L'armée, livre des hommes}

En consignant les récits de vie des vieux grognards, Vigny fait plusieurs choses à la fois. Il raconte l'initiation de son narrateur vers une nouvelle forme de socialité, celle de lécriture romanesque et poétique. Il introduit aussi son lecteur aux modalités d'une altérité à laquelle il n'aurait pas accès autrement, lui permettant d'avoir, lui aussi, la même expérience initiatique. L'écoute des récits oraux se redouble par une lecture des récits écrits. Le su prend forme dans le lu. Le narrateur extradiégétique est là pour surmonter la contradiction entre la tradition et l'écriture. Il structure alors un deuxième niveau expérientiel. La tragique relation entre la coutume militaire et les destins individuels est un de ces drames de la pensée dont nous parlions en introduction et le choc entre la matérialité de la lettre et l'esthétique de la mémoire structure ici cette idée philosophique.

Vigny se pose alors lui-même en passeur de symbole à l'image des conteurs qu'il met en scène. Pour permettre à son lecteur de s'élever à son tour, Vigny ne tranche pas dans cette querelle des modernes et des anciens. Il laisse à son lecteur la possibilité de vivre cette expérience de l'altérité comme il le fit lui-même auparavant. 
Le processus des récits enchâssés invite à l'interaction critique entre les paliers narratifs : la posture du jeune narrateur face aux récits de ses maitres peut être lue comme une mise en abyme de la lecture critique auquel invite Vigny. Il s'approprie et intègre leurs histoires tout en conservant une certaine autonomie, ce qu'il illustre bien lorsque, après l'histoire de "Laurette », il ne s'intéresse plus aux autres récits :

Il ne pensait pas à lui-même et, au bout d'un quart d'heure, il entama, sur le même ton, une histoire bien plus longue sur une campagne du maréchal Masséna, où il avait formé son bataillon en carré contre je ne sais quelle cavalerie. Je ne lécoutais pas, quoiqu'il séchauffât pour me démontrer la supériorité du cavalier sur le fantassin (Vigny, 1993, p. 718)

Ce genre de passage invite le lecteur à " déplacer son attention du texte vers le monde du texte » (Haubout, 2010, p. 214) et motive « une exhibition de la fabrique littéraire » (p. 215) qui pousse à une distanciation critique. Tout comme le narrateur choisit les histoires qui l'ont marqué, le lecteur peut se faire une opinion parce qu'il est lui-même passé au travers des mêmes étapes du récit-rite. «L'idée, écrit Vigny, se fera jour et prendra sa forme, comme fait tôt ou tard toute idée nécessaire. Dans l'état actuel, que de bons sentiments à conserver qui pourraient sélever encore par le sentiment d'une haute dignité personnelle ! »(1993, p. 724). Le lecteur a la possibilité de s'identifier ou de se distancier, voire de simplement conserver les éléments utiles, car la vérité se construit comme un ensemble fragmentaire : il dit bien écrire « des détails de mœurs » (1993, p. 683).

De cette leçon, il ne faut pas retenir de thèse. Le symbole vignyen permet d'accéder à une vérité négative qui, par contraste dialectique, hisse l'esprit. Révélatrice est, en ce sens, la structure par paliers du roman, où le je des grognards devient le je du narrateur pour ensuite monter jusqu'au récit-cadre et au lecteur. Dans la logique des savoirs narratifs et oraux, il suffit d'entendre un récit pour se l'approprier (Lyotard, 1979, p. 39) ; de même, subir l'initiation autorise à devenir initiateur. C'est pourquoi, au final, l'architecture du roman, où récit cadre et récit encadré, où narrateur intra et extradiégétique alternent sans cesse, nous apparaît être celle d'une expérience de maîtrise et de dépassement que les lecteurs méditeront avec une certaine liberté.

\section{RÉFÉRENCES}

Citoleux, M. (1924). Alfred de Vigny. Persistances classiques et affinités étrangères. Paris : Librairie ancienne Champion.

Cnockaert, V., Privat, J.-M. et Scarpa, M. (2011). Présentation : situation et émergence de l'ethnocritique. Dans V. Cnockaert, J.-M. Privat et M. Scarpa, L'Ethnocritique de la littérature (p. 1-9). Québec: Presses de l'Université du Québec.

Foucault, M. (2001). Dits et écrits, t. II. Paris : Gallimard. 
Hautbout, I. (2010). La construction d'un lecteur actif dans les romans de Vigny. Dans I. Haubout et M.-F. Melmoux-Montaubin (dir.), Alfred de Vigny romancier (p. 207-224). Amiens : Encrage Université.

Haubout, I. et Melmoux-Montaubin M.-F. (2010). Alfred de Vigny romancier : explorations critiques. Dans I. Haubout et M.-F. Melmoux-Montaubin (dir.), Alfred de Vigny romancier (p. 11-20). Amiens : Encrage Université.

Jarry, A. (1993). Vigny philosophe. Cahiers de l'Association internationale des études françaises, 45, 179-194.

Jarry, A. (1998). Alfred de Vigny. Étapes et sens du geste littéraire. Lecture psychanalytique. Genève : Droz.

Jarry, A. (2010). L'écriture comme génératrice de sens dans l'œuvre romanesque d'Alfred de Vigny. Dans I. Haubout et M.-F. Melmoux-Montaubin (dir.), Alfred de Vigny romancier (p. 73-88). Amiens : Encrage Université.

Kern, É. (2010). Le roman chez Vigny, une « sorte de poème épique » ? Dans I. Haubout et M.-F. Melmoux-Montaubin (dir.), Alfred de Vigny romancier (p. 159-176). Amiens : Encrage Université.

Lyotard, J.-F. (1979). La Condition postmoderne. Rapport sur le savoir. Paris : Minuit.

Ozwald, T. (2010). Servitude et grandeur militaires : un roman en devenir ? Dans I. Haubout et M.-F. Melmoux-Montaubin (dir.), Alfred de Vigny romancier (p. 119-139). Amiens : Encrage Université.

Privat, J.-M. (2017). Le chapeau de l'arpenteur. Polylogie, dialogie, hétérophonie. Dans S. Ménard et J.-M. Privat. À l'œuvre, l'œuvrier (p. 47-84). Nancy : Presses universitaires de Nancy-Université de Lorraine.

Rauch, A. (2000). La Crise de l'identité masculine (1789-1914). Paris : Hachette.

Saint-Gérard, J.-P. (2016). Alfred de Vigny et le monde des signes. Les deux parts du sens et la rencontre avec son lecteur. Dans I. Hautbout (dir.), Alfred de Vigny et le romantisme (p. 103-121). Paris : Classiques Garnier.

Scarpa, M. (2011). Le personnage liminaire. Dans V. Cnockaert, J.-M. Privat et M. Scarpa, L'Ethnocritique de la littérature (p. 177-189). Québec: Presses de l'Université du Québec.

Sohn, A.-M. (2009). "Sois un homme!" La Construction de la masculinité au XIX $X^{e}$ siècle, Paris : Seuil.

Van Gennep, A. (1981). Les Rites de passage, Paris : Picard.

Vigny, A. (1949). CEuvres complètes, t. II. Paris : Gallimard.

Vigny, A. (1986). Euvres complètes, t. I. Paris : Gallimard.

Vigny, A. (1993). CEuvres complètes, t. II. Paris : Gallimard.

RÉSUMÉ : Pendant son passage dans l'armée, Vigny a constaté la lente et inévitable disparition des vieux grognards. Ces hommes au caractère antique lui apparaissaient à la fois archaïques et nobles, c'est pourquoi, avec Servitude et grandeur militaires, il cherche à la fois à critiquer l'institution militaire et à conserver les enseignements de ces vétérans. Ces hommes sont présentés comme des maîtres à penser d'une nature particulière, ils sont à la fois des exemples à suivre et à dépasser. Suivant la logique des rites initiatiques, nous proposons une lecture ethnocritique de la poétique 
vignyenne qui s'attarde à reconstruire l'architecture narrative d'une expérience pédagogique. Celle-ci se structure autour de la présence de personnages liminaires servant de passeurs. Nous nous penchons ensuite sur l'appropriation critique de ces enseignements par le narrateur, appropriation que nous lisons comme une mise en abyme de l'attitude critique auquel Vigny convie ses lecteurs. Nous faisons ainsi ressortir la visée formatrice de l'écriture romanesque de l'écrivain romantique.

Mots-clés : Alfred de Vigny, Servitude et grandeur militaires, ethnocritique, passeur de symbole, personnage liminaire, rite de passage

\section{The army or the book of men: symbol transmitters and liminal characters in Alfred de Vigny's Servitude et grandeur militaires}

ABSTRACT: During his time in the army, Vigny noticed the slow and inevitable disappearance of the old grumblers. For him, these antique men are both archaic and noble, which is why, with Servitude et grandeur militaires, he tries to criticize the military institution, and record the teachings of these veterans simultaneously. These men represent a particular type of master, as their teachings must be both received and surpassed. Following the logic of rites of passage, we propose an ethnocritic reading of Vigny's poetic, and try to reconstruct the narrative architecture of a pedagogical experience. We believe the latter is organized around the presence of liminal characters which serve as transmitters. We then study the narrator's critical appropriation of these teachings, which we read as a mise en abyme of the critical attitude to which Vigny invites his readers. This way, we bring out the formative value of the fiction of that romantic writer.

Keywords: Alfred de Vigny, Servitude et grandeur militaires, ethnocritic, symbol transmitter, liminal character, rite of passage 